\title{
Probing trace levels of prometryn solutions: from test samples in the lab toward real samples with tap water
}

\author{
Rafael J. G. Rubira ${ }^{1}$ Sabrina A. Camacho ${ }^{1} \cdot$ Pedro H. B. Aoki $^{1,2}$. \\ Fernando V. Paulovich ${ }^{3} \cdot$ Osvaldo N. Oliveira Jr. ${ }^{2} \cdot$ Carlos J. L. Constantino $^{1}$
}

Received: 1 August 2015/Accepted: 27 November 2015/Published online: 17 December 2015

(C) The Author(s) 2015. This article is published with open access at Springerlink.com

\begin{abstract}
Growing food demand has been addressed by protecting crops from insects, weeds, and other organisms by increasing the application of pesticides, thus increasing the risk of environmental contamination. Many pesticides, such as the triazines, are poorly soluble in water and require trace detection methods, which are normally achieved with high-cost sophisticated chromatography techniques. Here, we combine surface-enhanced Raman scattering (SERS) with multidimensional projection techniques to detect the toxic herbicide prometryn in ultrapure, deionized, and tap waters. The SERS spectra for prometryn were recorded with good signal-to-noise ratio down to $5 \times 10^{-12} \mathrm{~mol} / \mathrm{L}$ in ultrapure water, approaching singlemolecule levels, and $5 \times 10^{-9} \mathrm{~mol} / \mathrm{L}$ in tap water. The latter is one order of magnitude below the threshold allowed for drinking water. In addition to providing a fingerprint of prometryn molecules at low concentrations, SERS is advantageous compared to other methods since it does not require pretreatment or chemical separation. The multidimensional projection methods and the detection procedure with SERS are entirely generic, and may be
\end{abstract}

Electronic supplementary material The online version of this article (doi:10.1007/s10853-015-9628-2) contains supplementary material, which is available to authorized users.

Rafael J. G. Rubira

rafael.gon.fis@gmail.com

1 Faculdade de Ciências e Tecnologia, UNESP Univ Estadual Paulista, Presidente Prudente, SP 19060-900, Brazil

2 São Carlos Institute of Physics, University of São Paulo, CP 369, São Carlos, SP 13560-970, Brazil

3 Institute of Mathematical Sciences and Computing, University of São Paulo, CP 668, São Carlos, SP 13560-970, Brazil extended to any other pesticide or water contaminants, thus allowing environmental control to be potentially low cost if portable Raman spectrophotometers are used.

\section{Introduction}

The growing demand for food in conjunction with competition from international markets forces the agribusiness to increasingly resort to agrochemicals, as is the case of Brazil where the sales of pesticides have increased dramatically to position the country as the largest consumer in the world, ahead of the USA [1]. The lack of criteria for controlling the use of pesticides represents a severe environmental issue due to contamination of plants, soil, and groundwater. Furthermore, the easy dispersion of pesticides in the atmosphere and oceans can rapidly reach global scale contamination [2]. According to the World Health Organization (WHO), millions of people are poisoned by these substances every year, leading to approximately 220,000 deaths [3]. From the various types of herbicides, triazine derivatives are prominent for their use in the pre- and post-emergent control of weed seeds in a variety of crops, including corn, sugar cane, sorghum, pineapple, banana, coffee, and grapes [4]. The triazine derivatives and their degradation products are toxic and nonbiodegradable, which can lead to environmental contamination. Particularly relevant is contamination in groundwater and drinking water [5]. Prometryn is one of the most used herbicides of the triazine family, whose maximum concentration allowed in drinking water is $23.2 \mu \mathrm{g} / \mathrm{L}\left(9.62 \times 10^{-8} \mathrm{~mol} / \mathrm{L}\right.$ or $\left.23.2 \mathrm{ppb}\right)$, according to regulations from the United States Environmental Protection Agency (USEPA) [6]. 
Only a few analytical methods are available that can reach such level of dilution, most of which involve chromatography experiments [7, 8]. Electrochemical techniques are also employed in detecting prometryn, although electrochemical sensors based on mercury electrodes [911] have negative environmental implications. Motivated by the need to develop sensitive and selective methods to monitor residual amounts of prometryn, in this study we combine the highly sensitive and selective surface-enhanced Raman scattering (SERS) [12] with advanced computational data analysis in order to detect prometryn below the threshold allowed for drinking water. SERS is a vibrational technique in which signal enhancement of target molecules is achieved due to localized surface plasmon resonance (LSPR) excited using metallic nanostructures [13-15]. Basically, the incident electric field $\left(E_{\text {inc }}\right)$, from the probing laser, interacts with the metallic nanoparticles and induces a collective oscillation of the electronic metal cloud, which is able to sustain LSPR. In terms of electric field, the nanoparticle excitation induces an electric field $\left(E_{\mathrm{p}}\right)$ in its surroundings, leading to a local electric field ( $\left.E_{\text {local }}\right)$ given by the sum $E_{\text {local }}=E_{\text {inc }}+E_{\mathrm{p}}$, which is much larger than $E_{\text {inc }}$ itself and oscillates with the same frequency of the probing laser. When the target molecule is close enough to the nanoparticle, $E_{\text {local }}$ polarizes the target molecule, inducing a molecular dipole $\left(p_{1}\right)$. The electric field irradiated by $\mathrm{p}_{1}$ can also polarize the metallic nanoparticle, inducing a dipole in the nanoparticles $\left(p_{2}\right)$, which oscillates with the same frequency of $p_{1}$ (Raman frequency). The intense local electric field ( $\left.E_{\text {local }}\right)$ is proportional to $\mathrm{E}^{4}$ and can achieve an enhancement factor from $10^{3}$ to $10^{6}$ in average and up to $10^{10}$ in the so-called "hot spots" (interstices of AgNPs aggregates where extremely high density of electric field is found) [13-16].

The samples used in the experiments included prometryn solutions in ultrapure, deionized, and tap waters, in which the latter mimics the conditions found in real samples.

\section{Materials and methods}

\section{Reagents}

The reagents silver nitrate $\left(\mathrm{AgNO}_{3}, \mathrm{MM}=169.88 \mathrm{~g} / \mathrm{mol}\right)$, hydroxylamine hydrochloride $\left(\mathrm{NH}_{2} \mathrm{OH} \cdot \mathrm{HCl}, \quad \mathrm{MM}=\right.$ $69.49 \mathrm{~g} / \mathrm{mol})$, and sodium hydroxide $(\mathrm{NaOH}, \mathrm{MM}=$ $40.00 \mathrm{~g} / \mathrm{mol})$ were acquired from Sigma-Aldrich. Prometryn $\left(\mathrm{C}_{10} \mathrm{H}_{19} \mathrm{~N}_{5} \mathrm{~S}, \mathrm{MM}=241.36 \mathrm{~g} / \mathrm{mol}\right)$, purity $=98.8 \%$, was purchased from Fluka Analytical. All the chemicals were used without further purification. Ultrapure water with resistivity of $18.2 \mathrm{M} \Omega \mathrm{cm}$ and $\mathrm{pH} 5.6$, acquired from a Milli-Q system (model Simplicity), was used to prepare the Ag colloid and prometryn stock solution. Deionized tap water with resistivity of $0.3 \mathrm{M} \Omega \mathrm{cm}$, acquired from a deionization system (model SP-050C), was also used to prepare a prometryn stock solution. The neutralization process removes nitrates, calcium, magnesium, cadmium, barium, lead, and some forms of radium from the water [17].

\section{Synthesis of Ag colloid by hydroxylamine reduction}

The Ag colloid (AgNPs) obtained by hydroxylamine reduction was synthesized according to the methodology described by Leopold and Lendl [18]. The synthesis consisted in adding $4.5 \mathrm{~mL}$ aqueous solution of $\mathrm{NaOH}$ $0.1 \mathrm{~mol} / \mathrm{L}$, at room temperature, into $5 \mathrm{~mL}$ of $\mathrm{NH}_{2} \mathrm{OH} \cdot \mathrm{HCl}$ $43.3 \mathrm{mmol} / \mathrm{L}$ solution. This solution was added to $90 \mathrm{~mL}$ of $\mathrm{AgNO}_{3}$ at $1.2 \mathrm{mmol} / \mathrm{L}$, under stirring, thus yielding a AgNPs colloidal dispersion with a final concentration of ca. $1.0 \mathrm{mmol} / \mathrm{L}$ [18]. The UV-Vis spectrum and SEM image of the Ag colloid are shown in Figure S1 in the Supplementary Material, in which results from zeta potential, size and shape of the AgNPs are discussed.

\section{UV-Vis absorption, SEM, zeta potential, and Raman scattering}

The UV-Vis absorption spectrum of the Ag colloid was recorded using a Varian spectrophotometer, model Cary 50, from 190 to $1100 \mathrm{~nm}$. Scanning electron microscope (SEM) images were taken with a Carls Zeiss equipment, EVO LS15 model (Laboratory LabMMEV at FCT/ UNESP). The zeta potential was determined using a ZetaSizer3000 HS. The Micro-Raman analysis was performed using a micro-Raman Renishaw spectrograph, model in-Via, equipped with a Leica microscope, where a $\times 50$ objective lens allows collecting spectra with ca. $1 \mu \mathrm{m}^{2}$ spatial resolution. The spectrograph contains a chargecoupled device (CCD) detector, laser line at $633 \mathrm{~nm}, 1800$ grooves/mm grating with additional edge filters. In all measurements, the Raman scattering and SERS spectra were acquired with spectral acquisition times of $10 \mathrm{~s}$ and laser power within the $\mu \mathrm{W}$ range at the sample.

\section{Solutions for SERS measurements}

Three prometryn stock solutions at $1 \times 10^{-4} \mathrm{~mol} / \mathrm{L}$ were prepared by dissolving $6.0 \mathrm{mg}$ of prometryn in $250 \mathrm{~mL}$ of ultrapure, deionized, and tap waters, under sonication. These stock solutions were diluted in Ag colloid to achieve SERS. The final solution concentrations of prometryn were $5 \times 10^{-5}, 5 \times 10^{-6}, 5 \times 10^{-7}, 5 \times 10^{-8}, 5 \times 10^{-9}$, $5 \times 10^{-10}, 5 \times 10^{-11}$, and $5 \times 10^{-12} \mathrm{~mol} / \mathrm{L}$. In order to acquire SERS spectra, a small droplet of prometryn 
solution (diluted in Ag colloid) was placed in a holder under the microscope and the laser focus was adjusted onto the air/water interface. Usually, several spectra were recorded from 2 different drops until getting at least 8 spectra with suitable signal/noise ratio for each drop. For each concentration ( 2 drops), the measurements were performed in a total of approximately $2 \mathrm{~h}$, for which the solvent evaporation (water) was minimal. Fig. 1 illustrates the procedure adopted in the SERS measurements.

\section{Data analysis}

The SERS spectra were analyzed using a multidimensional projection technique, in which data from a multidimensional space can be projected onto a $2 \mathrm{D}$ space creating a plot with maximum preservation of similarity relationships. Formally, the data in the original space are represented by $X=\left\{x_{1}, x_{2}, \ldots, x_{n}\right\}$, and $\delta\left(x_{i}, x_{j}\right)$ is defined as the distance between two data instances $i$ and $j$. The 2D plot is created by projecting them onto the plane with graphical markers represented by $Y=\left\{y_{1}, y_{2}, \ldots, y_{n}\right\}$, with the positions on the 2D plot being determined in an optimization procedure using an injective function $\mathrm{f}: \mathrm{X} \rightarrow \mathrm{Y}$ that minimizes $I \delta\left(x_{i}\right.$, $\left.x_{j}\right)-d\left(\mathrm{f}\left(x_{i}\right), \mathrm{f}\left(y_{j}\right)\right) \mid \approx 0, \forall x_{i}, x_{j} \in \mathrm{X}[19]$, where $\mathrm{d}\left(y_{\mathrm{i}}, y_{\mathrm{j}}\right)$ is the distance function on the projected plane. The flexibility of this optimization approach arises from the availability of several cost (or error) functions used for placing the

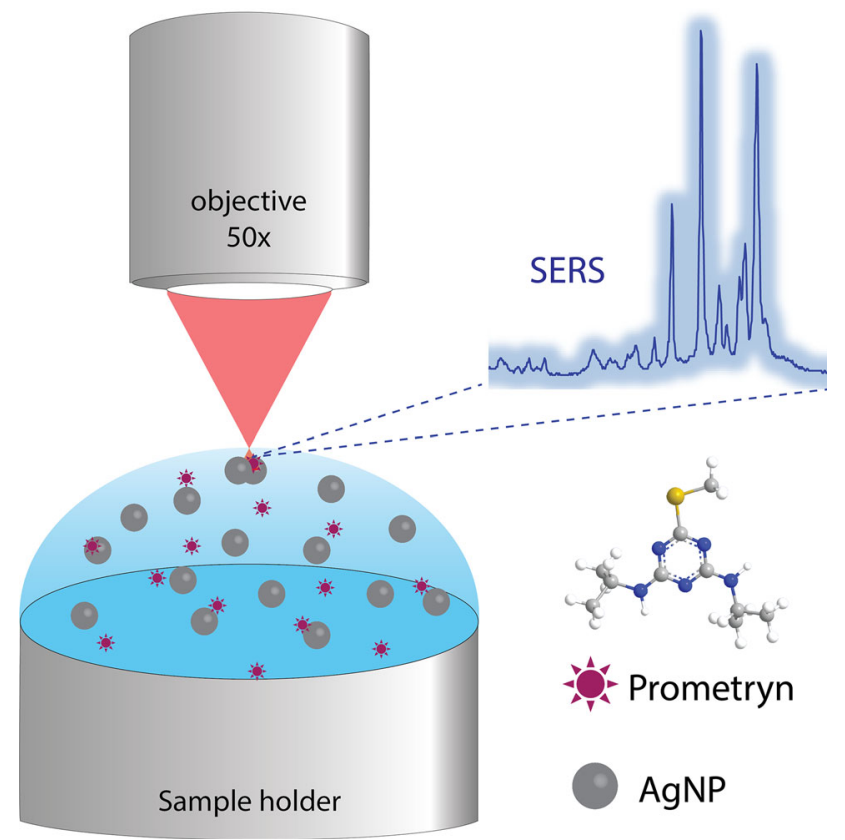

Fig. 1 Schematic procedure for the SERS measurements. The prometryn molecular structure is represented in $3 \mathrm{D}$ where the atoms are distinguished by the colors: $\mathrm{H}$ (white), $\mathrm{C}$ (gray), $\mathrm{N}$ (blue), and $\mathrm{S}$ (yellow) (Color figure online) graphical markers on the $2 \mathrm{D}$ plot. Here we used the socalled Interactive Document Map (IDMAP) [20], whose function is defined as follows:

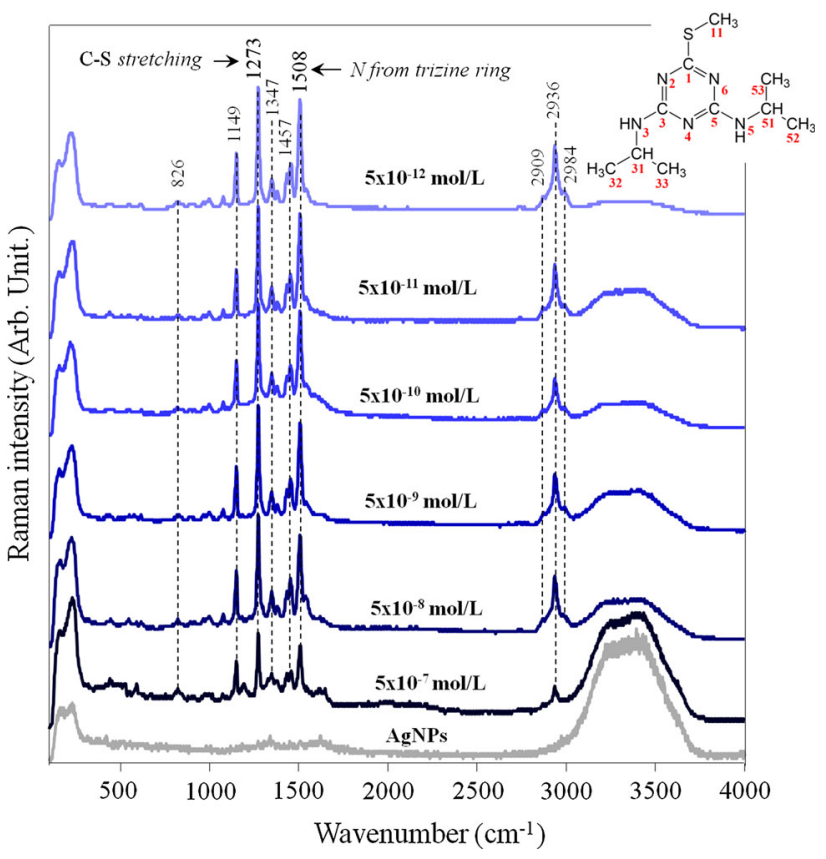

Fig. 2 SERS spectra (raw data) of prometryn ultrapure water solutions diluted in $\mathrm{Ag}$ colloid at $5 \times 10^{-7}, 5 \times 10^{-8}, 5 \times 10^{-9}$, $5 \times 10^{-10}, 5 \times 10^{-11}$, and $5 \times 10^{-12} \mathrm{~mol} / \mathrm{L}$. The Raman intensity ( $Y$-axis) is normalized. An Ag colloid spectrum is given as reference (control) at the bottom

Table 1 Vibrational assignments of prometryn characteristic bands [24, 25]

\begin{tabular}{ll}
\hline $\begin{array}{l}\text { Prometryn } \\
\left(\mathrm{cm}^{-1}\right)\end{array}$ & Assignments \\
\hline 703 & (C1-S stretching) [24, 25] \\
826 & (Ring breathing) [24] \\
902 & (Ring breathing) [24] \\
970 & (Ring breathing) [24] \\
1149 & (C-S stretching); (isopropyl group deformation) [24, 25] \\
1176 & (C-S stretching) [24, 25] \\
1273 & (Plane deformation of the ring); (C-S stretching); (N-C- \\
& H deformation); (C-C-H deformation) [24, 25] \\
1310 & (Plane deformation of the ring); (N-C-H deformation); \\
& (C-S stretching); (C-C-H deformation) [24, 25] \\
1347 & (S-CH symmetric bending + lateral chains C-C-H \\
& deformation) [24, 25] \\
1457 & (CH ${ }_{3}$ deformation on the sulfanyl group) [24, 25] \\
2760 & (C-H stretching); (H-C-C bending) [24] \\
2873 & (C-H stretching of the CH ${ }_{3}$ groups) [24] \\
2909 & (C51-H + C52-H + C53-H stretching) [24] \\
2936 & (C31-H + C32-H + C33-H stretching) [24] \\
2984 & (C31-32-H symmetric and antisymmetric stretchings) \\
& [24] \\
\hline &
\end{tabular}


$S_{\text {IDMAP }}=\frac{\delta\left(x_{i}, x_{j}\right)-\delta_{\min }}{\delta_{\max }-\delta_{\min }}-d\left(y_{i}, y_{j}\right)$

where $\delta$ and $d$ are the distance functions defined above and $\delta_{\min }$ and $\delta_{\max }$ are the minimum and maximum distances between the samples.

\section{Results and discussion}

\section{Probing prometryn in ultrapure water solutions}

The SERS effect was applied in detecting highly diluted solutions of prometryn in order to include the maximum value allowed for drinking water $\left(10^{-8} \mathrm{~mol} / \mathrm{L}\right)$ according to regulation by the United States Environmental Protection

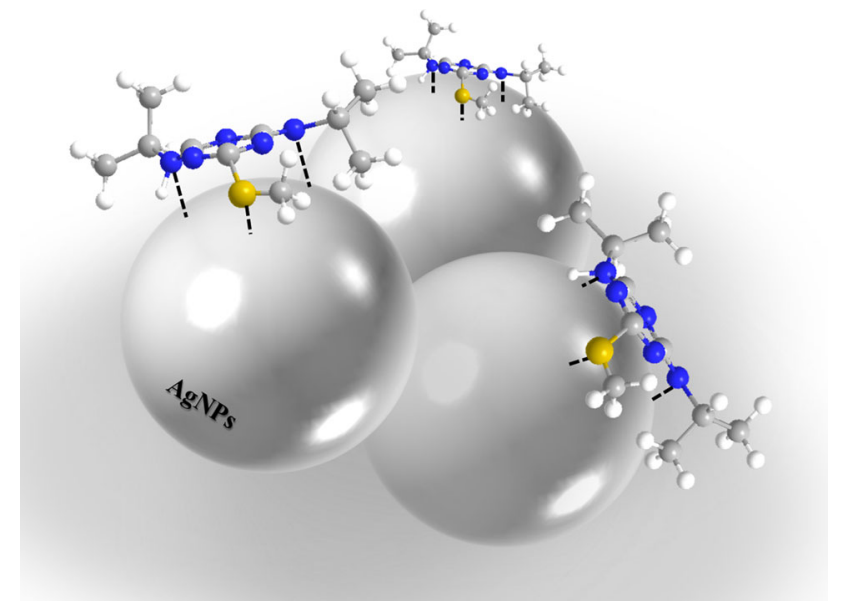

Fig. 3 Schematic representation of the prometryn adsorption onto AgNPs, which is believed to occur via interactions of their N and C-S groups, with the ring lying parallel to the AgNPs
Agency (USEPA) [6]. The solution concentration plays a role because aggregation of prometryn molecules may hinder adsorption onto the Ag colloid [21], thus leading to smaller SERS enhancement. Figure S2 in the Supplementary Material shows SERS spectra collected at relatively high concentrations of $5 \times 10^{-5}$ and $5 \times 10^{-6} \mathrm{~mol} / \mathrm{L}$. Despite the low signal/noise ratio, these SERS spectra were found in all the measurements for these high concentrations. In contrast, intense, well-defined vibrational bands are seen in Fig. 2 for solutions from $5 \times 10^{-7} \mathrm{~mol} / \mathrm{L}$ down to $10^{-12} \mathrm{~mol} / \mathrm{L}$. Two main points should be highlighted here: (i) the spectral acquisition from $5 \times 10^{-7}$ down to $10^{-12} \mathrm{~mol} / \mathrm{L}$ is not straightforward because only the molecules adsorbed at the interstices of AgNPs aggregates ("hot spots") will give rise to measurable signals; (ii) the SERS spectra in Fig. 2 are normalized and a linear increase of the SERS signal with concentration was not observed at this range of concentration. Obtaining analytical curves using SERS (e.g., band intensity vs. prometryn concentration) is not straightforward because band intensity depends on several factors, in addition to the analyte concentration. For example, the SERS intensity may fluctuate owing to the movement of metallic nanoparticlestarget molecules in and out of the volume probed by the laser. It also depends on the size, shape, and aggregation of the metallic nanoparticles, which is the reason why there are only a few works in the literature with attempts to correlate band intensity with analyte concentration [22].

The spectra taken from neat Ag colloid, shown in Figure S3 in the Supplementary Material, are used as reference (control). Since only the signal expected from the Ag colloid was observed in these samples, one may be sure of the low level of impurities. The main vibrational bands in the SERS spectra for the solutions in Fig. 2 are highlighted by dotted lines, with the assignments given in Table 1 .
Fig. 4 IDMAP

multidimensional projection grouping the results by different concentrations of prometryn in ultrapure water solutions. Each circle represents a whole SERS spectrum. The closer the circles, the more similar the SERS spectra

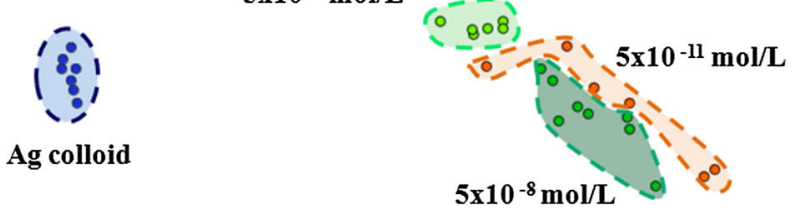

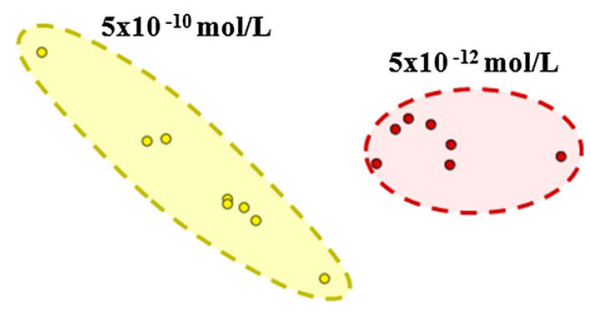


According to the selection rules for SERS [13-16, 23], vibrational modes with dipole moment perpendicular to $\mathrm{Ag}$ surface (consequently, parallel to the electric field) result in a high intensity of the SERS signal. Larger enhancements are found for bands assigned to the vibrational modes involving the ring, within the $1500-1100 \mathrm{~cm}^{-1}$ region [24], suggesting that the aromatic $\mathrm{N}$ atoms play a role in the prometryn adsorption onto the AgNPs. Furthermore, the largest enhancement of the band at $1273 \mathrm{~cm}^{-1}$ (inplane deformation of the ring mixed with the stretching of $\mathrm{C}-\mathrm{S}[24,25])$ indicates that prometryn molecules are preferentially adsorbed onto AgNPs through the C-S groups, as depicted in Fig. 3. Besides, we highlight the band at $970 \mathrm{~cm}^{-1}$ (ring breathing), which is the most intense in the powder spectrum but decreased drastically in the SERS spectrum. This suggests that the prometryn molecules have their triazinic ring positioned parallel to the AgNPs surface (selection rules [13]). This conclusion is supported by the SERS experimental results from Bonora et al. [24] working with atrazine, simetryn, and prometryn herbicides, and by theoretical calculations of Benassi et al. [25].

A visual inspection of Fig. 2 allows one to infer whether it is possible to detect the different prometryn concentrations. In order to demonstrate this distinction ability when many samples are compared, one has to resort to statistical or computational methods for analyzing the data. Here we confirmed such distinction ability by treating the SERS spectra shown in Fig. 2 with the IDMAP multidimensional projection technique, whose results are given in Fig. 4. Details of multidimensional projection techniques can be found in Paulovich et al. [26] and Oliveira et al. [27]. Basically, each circle in the plot represents a whole spectrum, and the closer the circles, the more similar the SERS spectra. Samples with distinct prometryn concentrations are clustered apart from each other, indicating that SERS can easily distinguish samples even down to $10^{-12} \mathrm{~mol} / \mathrm{L}$. A larger dispersion of the data is seen for prometryn solutions at $10^{-10}, 10^{-11}$, and $10^{-12} \mathrm{~mol} / \mathrm{L}$, which is a direct consequence of fluctuations on the SERS spectra affecting bandwidth, band shape, Raman shift, and absolute and relative intensities for highly diluted solutions. This is typical of the unique behavior observed as single-molecule limits are approached, revealing the breakdown of ensemble averaging SERS spectra and the local changes of molecular environment [13, 28]. Indeed, the number of prometryn molecules per picoliter $\left(10^{-12} \mathrm{~L}\right)$ at $5 \times 10^{-12}$ $\mathrm{mo} / \mathrm{L}$ is estimated to be three. The picoliter scale is the order of magnitude of the volume probed by a laser in single-molecule experiments [29, 30].

The smallest concentration tested here is the lowest ever detected for prometryn solutions, approaching singlemolecule levels. In fact, the use of SERS for prometryn in the literature has never gone below $10^{-4} \mathrm{~mol} / \mathrm{L}$ [24]. Prometryn detection with chromatography techniques has reached limits from $10^{-6}$ to $10^{-10} \mathrm{~mol} / \mathrm{L}[31,32]$ while the differential pulse polarographic method led to detection of atrazine, prometryn, and simazine herbicides down to $8 \times 10^{-8} \mathrm{~mol} / \mathrm{L}$ [7]. Oliveira-Brett et al. [33] reported an electrochemical biosensor to investigate the interactions
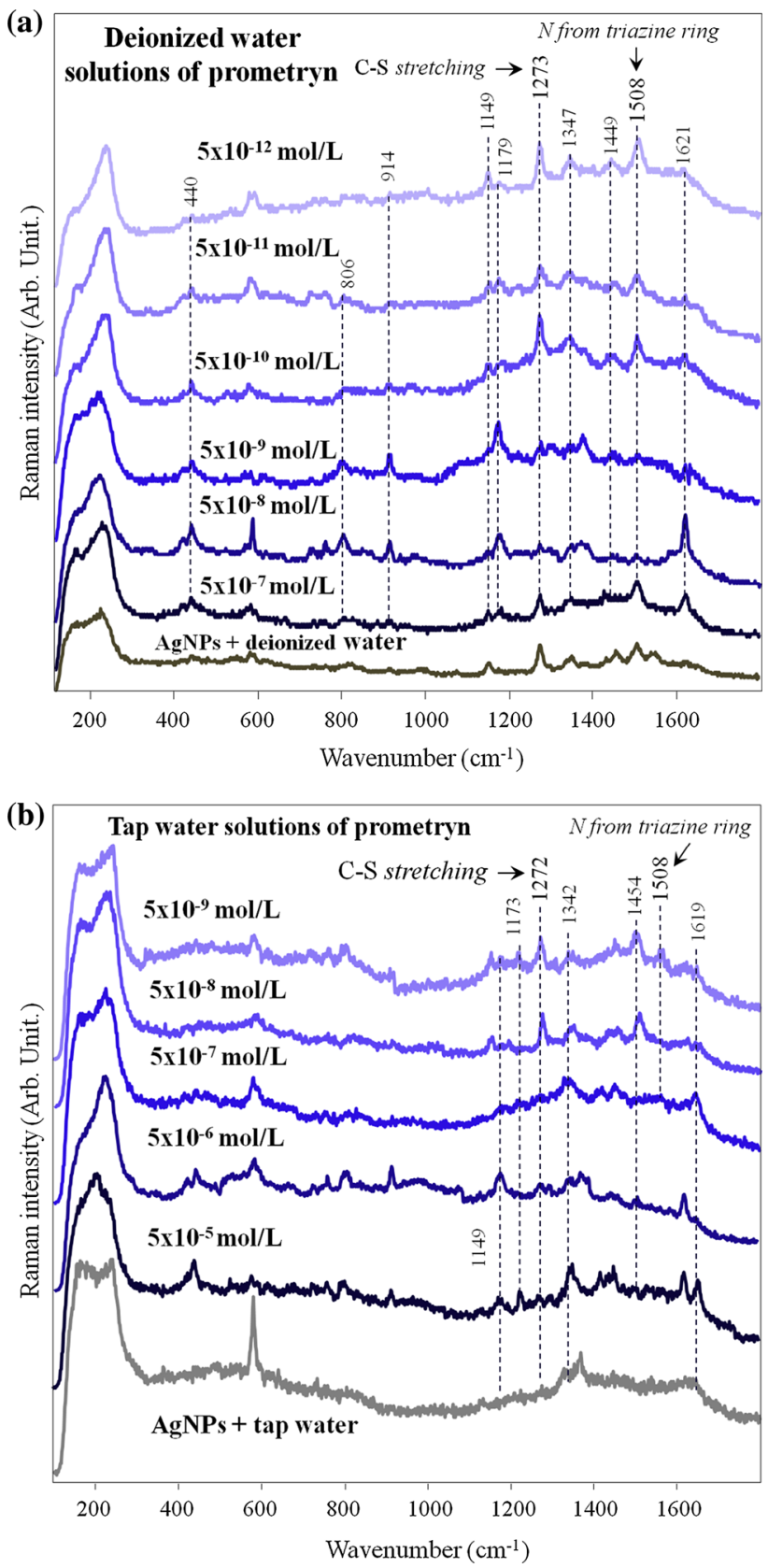

Fig. 5 SERS spectra (raw data) collected for: a deionized water solutions of prometryn diluted in Ag colloid at $5 \times 10^{-7}, 5 \times 10^{-8}$, $5 \times 10^{-9}, 5 \times 10^{-10}, 5 \times 10^{-11}, 5 \times 10^{-12} \mathrm{~mol} / \mathrm{L}$ and $\mathbf{b}$ tap water solutions of prometryn at $5 \times 10^{-5}, 5 \times 10^{-6}, 5 \times 10^{-7}, 5 \times 10^{-8}$, and $5 \times 10^{-9} \mathrm{~mol} / \mathrm{L}$. The Raman intensity ( $Y$-axis) is normalized. $\mathrm{Ag}$ colloid spectra containing deionized and tap water are given as reference (control) at the bottom of each figure 
between DNA and herbicides from the s-triazine group, achieving a detection limit of $5 \times 10^{-4} \mathrm{~mol} / \mathrm{L}$.

\section{Probing trace levels of prometryn in deionized and tap water solutions}

One important challenge in sensing experiments is to deal with real samples, as is the case of probing trace levels of prometryn in deionized and tap water solutions, which mimic the conditions prevailing in real samples. Figure 5a shows the SERS spectra collected for deionized water solutions of prometryn diluted in Ag colloid at $5 \times 10^{-7}$, $5 \times 10^{-8}, 5 \times 10^{-9}, 5 \times 10^{-10}, 5 \times 10^{-11}, \quad$ and
$5 \times 10^{-12} \mathrm{~mol} / \mathrm{L}$. The SERS spectra collected for tap water solutions of prometryn diluted in Ag colloid at $5 \times 10^{-5}, 5 \times 10^{-6}, 5 \times 10^{-7}, 5 \times 10^{-8}$, and $5 \times 10^{-9}$ $\mathrm{mol} / \mathrm{L}$ are displayed in Fig. 5b. Several Ag colloid spectra are given in the Supplementary Material (Fig. S4). The signal arising from the $\mathrm{Ag}$ colloid containing either deionized or tap water indicates the presence of impurities in the medium that might interfere in the SERS signal of the target molecule. Indeed, there is an overlap of bands between 1100 and $1621 \mathrm{~cm}^{-1}$ for prometryn and Ag colloid spectra, as shown in Fig. 5a, b.

The suitability of SERS to identify prometryn in deionized tap water solutions is more clearly visualized in
Fig. 6 IDMAP

multidimensional projection grouping the results by different concentrations of prometryn in deionized water solutions. Each circle represents a whole SERS spectrum. The closer the circles, the more similar the SERS spectra

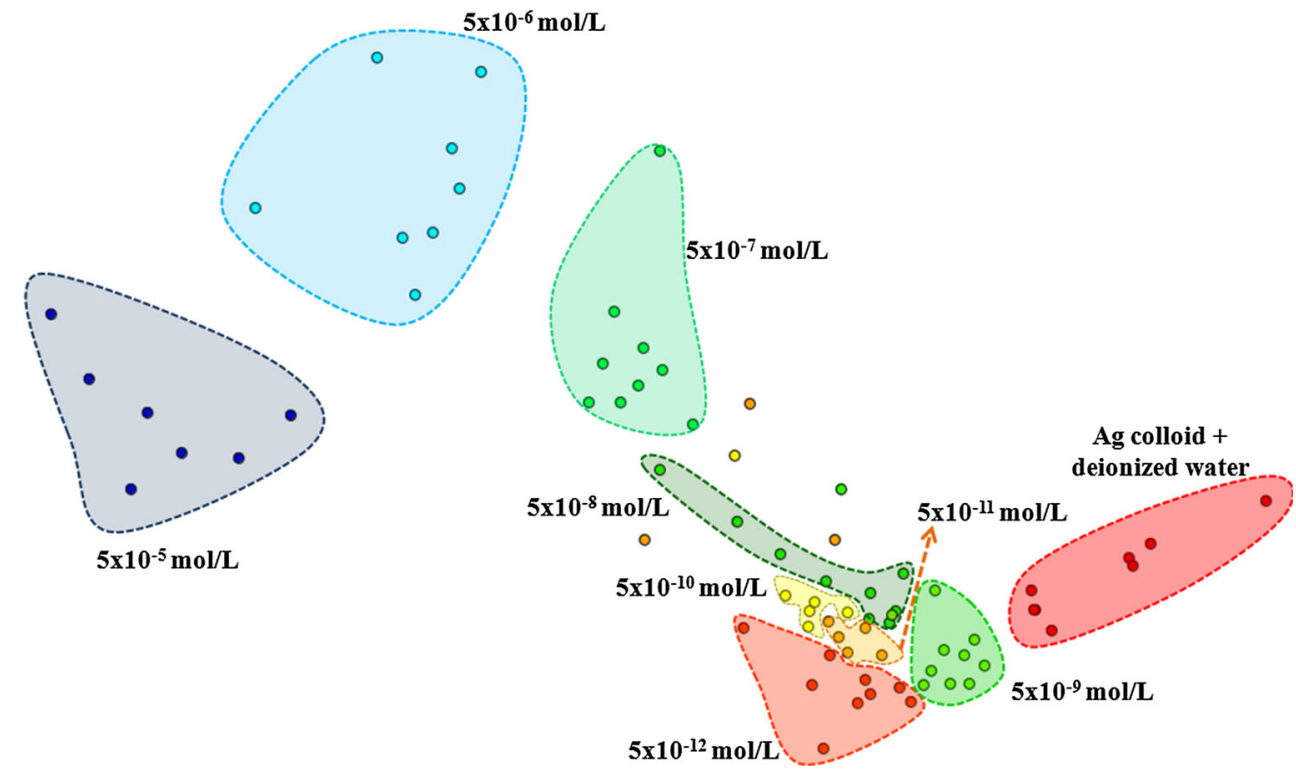

Fig. 7 IDMAP

multidimensional projection grouping the results by different concentrations of prometryn in tap water solutions. Each circle represents a whole SERS spectrum. The closer the circles, the more similar the SERS spectra
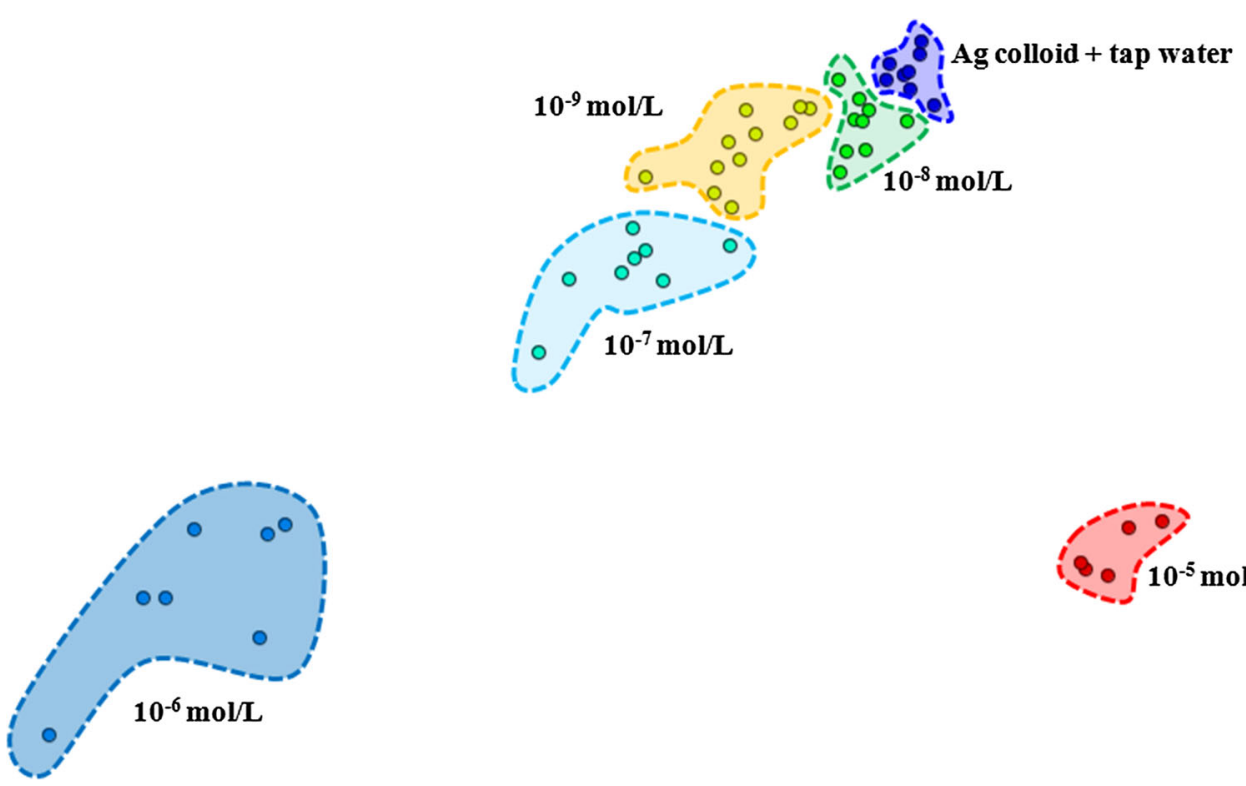
the 2D plot in Fig. 6 where the SERS spectra were treated using the IDMAP multidimensional technique. The results are grouped according to the similarity of the analyzed data. Prometryn concentrations at $5 \times 10^{-5}, 5 \times 10^{-6}$, $5 \times 10^{-7}, 5 \times 10^{-8}$, and $5 \times 10^{-9} \mathrm{~mol} / \mathrm{L}$ are clustered apart from each other. However, the clusters associated with more diluted prometryn solutions $\left(5 \times 10^{-10}\right.$, $5 \times 10^{-11}$, and $5 \times 10^{-12} \mathrm{~mol} / \mathrm{L}$ ) are lumped together and some overlap occurs. Hence, though the samples containing prometryn can be distinguished from deionized water-even down to $5 \times 10^{-12} \mathrm{~mol} / \mathrm{L}$ - they cannot be clearly separated among themselves.

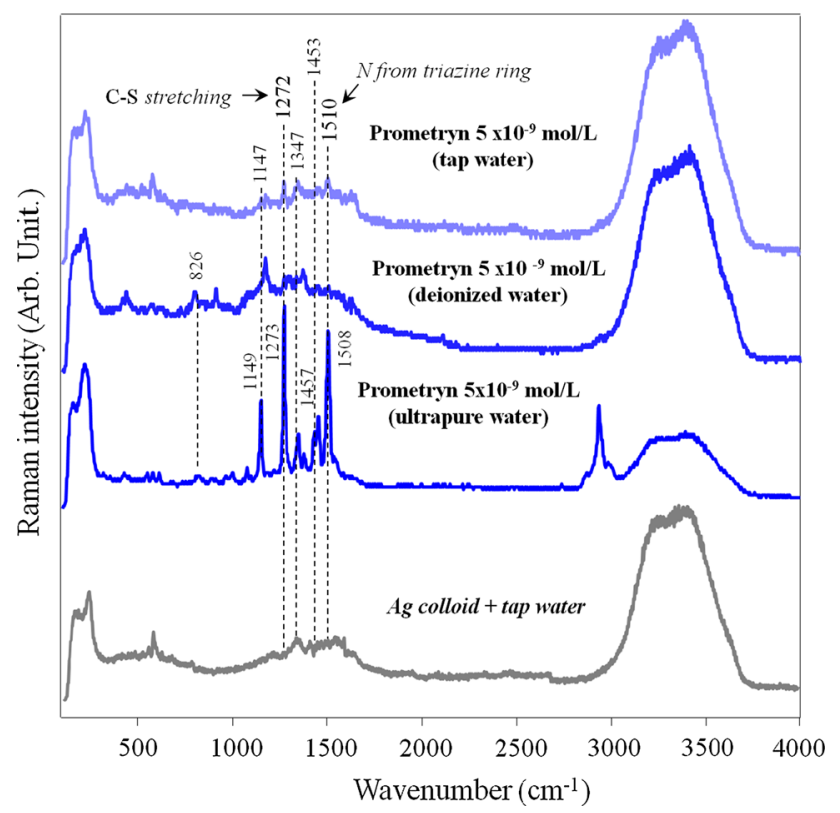

Fig. 8 SERS spectra of prometryn solutions in ultrapure $\left(5 \times 10^{-9}\right.$ $\mathrm{mol} / \mathrm{L})$, deionized $\left(5 \times 10^{-9} \mathrm{~mol} / \mathrm{L}\right)$, and tap water $\left(5 \times 10^{-9} \mathrm{~mol} / \mathrm{L}\right)$ compared with the reference spectrum (control) of the Ag colloid containing tap water
The distinguishing ability using SERS spectra is the poorest for prometryn solutions obtained from tap water, which should be expected due to its larger amount of impurities. Figure 7 shows the projection map of the data acquired for tap water solutions of prometryn only for concentrations starting at $10^{-9} \mathrm{~mol} / \mathrm{L}$. We omitted the data for lower concentrations, since their clusters would collapse into the cluster for the Ag colloid reference (control).

The SERS spectra of prometryn solutions prepared with ultrapure, deionized, or tap waters are shown in Fig. 8, besides the Raman spectrum of the Ag colloid containing tap water. The main vibrational bands in the SERS spectra for the solutions are highlighted by dotted lines (Fig. 8). The signal/noise ratio for the spectra collected in deionized and in tap water solutions of prometryn is not as high as in ultrapure water solutions, with the vibrational bands of prometryn being less defined (Fig. 8). The SERS signal is highly dependent on the distance between the metal nanoparticle surface and the target molecule [13], practically vanishing for distances larger than $150 \AA$. Therefore, impurities in deionized and in tap water may adsorb onto AgNPs and hinder the direct contact of prometryn molecules with the metal surface. The higher signal/noise ratio are found for the bands within $1500-1100 \mathrm{~cm}^{-1}$ region, assigned to the vibrational modes involving the ring [24]. The vibrational modes assigned to isopropyl groups between 2909 and $2984 \mathrm{~cm}^{-1}$ are no longer observed (see Fig. 8). Then, it seems that the triazine ring might also play a role in the adsorption onto the AgNPs surface for deionized and tap water.

The role of impurities is highlighted by the IDMAP projection in Fig. 9 in which the data are plotted from different Ag colloid references (ultrapure, deionized, and tap waters) and prometryn solutions at $10^{-9} \mathrm{~mol} / \mathrm{L}$ (ultrapure, deionized, and tap waters). It is clear that the projection technique (IDMAP) results show different patterns depending on the system analyzed. For instance, in general
Fig. 9 IDMAP

multidimensional projection grouping the results from the different Ag colloid references and prometryn solutions at $10^{-9}$ $\mathrm{mol} / \mathrm{L}$ in ultrapure, deionized, and tap water. Each circle represents a whole SERS spectrum. The closer the circles, the more similar the SERS spectra

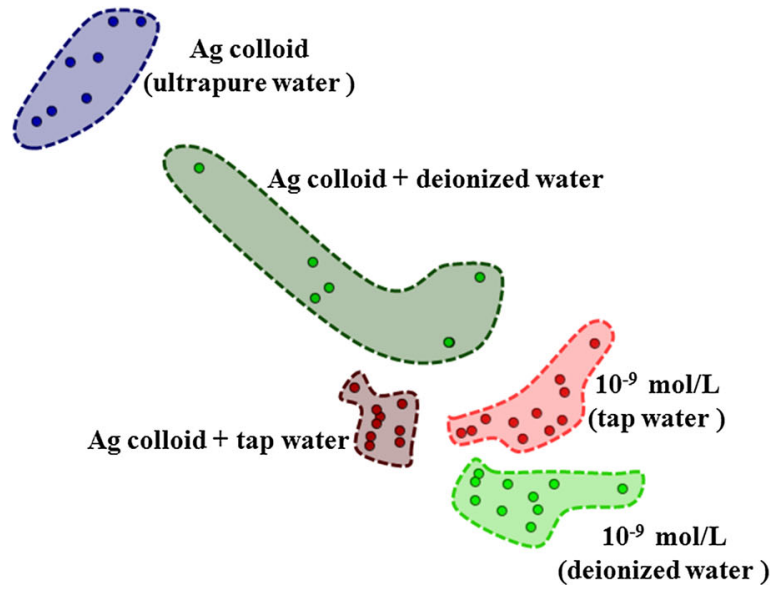

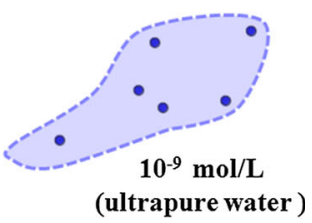


it is seen that the circles (within a cluster) are more spread for lower concentrations when prometryn is diluted in ultrapure water. On the other hand, considering the prometryn diluted in deionized or tap water, the circles (within a cluster) are more spread for higher concentrations. These opposite patterns could be understood considering that for ultrapure water (high purity medium), the addition of small amounts of impurity (prometryn) would be enough to lead to dramatic changes in the medium. For deionized or tap water (high impurity medium), the addition of small amounts of impurity (prometryn) would not be enough to interfere substantially in the medium because it is already impure. It is likely that a larger number of spectra will be required to understand the dispersion in the data for real samples, i.e., containing considerable amounts of impurities.

Therefore, even in the worst scenario represented by solutions made with tap water, treating the SERS data with IDMAP allows one to detect prometryn to concentrations one order of magnitude below the limit allowed for drinking water. This highlights the usefulness of projection techniques not only to handle the data but also to optimize the sensing performance. The performance of SERS-based detection is competitive with a few works in the literature for detection of pesticides in real samples, most of them based on extraction and size exclusion methods in chromatography techniques [8, 34-36]. Koeber et al. were able to detect triazine herbicides in river samples with a detection limit of $3.3 \times 10^{-10} \mathrm{~mol} / \mathrm{L}$ for simazine by combining column size exclusion and adsorption chromatography [31]. Djozan et al. reported detection of s-triazine herbicides in tap water with detection limit of $8.29 \times 10^{-8} \mathrm{~mol} / \mathrm{L}$ using gas chromatography [37]. Electrochemical techniques have also been applied to detect herbicides in solutions, foods, and real samples. Some limits of detection (LOD) reported were $1.7 \times 10^{-8} \mathrm{~mol} / \mathrm{L}$ for methidathion [38], $1.9 \times 10^{-11} \mathrm{~mol} / \mathrm{L}$ for paraquat in buffer or in potato extracts [39], and $8.9 \times 10^{-8} \mathrm{~mol} / \mathrm{L}$ for lindane in ultrapure water [40].

In conclusion, the combination of SERS with information visualization methods was successfully applied to detect prometryn not only in ultrapure water but also in tap water, which resembles real samples. The concentration detected is the lowest ever reported for prometryn in ultrapure water $\left(5 \times 10^{-12} \mathrm{~mol} / \mathrm{L}\right)$ and among the lowest for real samples of nonpurified water $\left(10^{-9} \mathrm{~mol} / \mathrm{L}\right)$. The prometryn detection in ultrapure water approached singlemolecule levels, which is promising since the experimental procedure for the measurement is carried out with the herbicide being directly detected in the sample, with no pretreatment or chemical separation. The lower SERS signal/noise ratio for prometryn in tap water is related to impurities in the samples that may hinder adsorption of the analyte molecules onto the colloidal AgNPs. The lowest prometryn concentration detected $\left(10^{-9} \mathrm{~mol} / \mathrm{L}\right)$ in tap water is still one order of magnitude below the threshold for drinking water. Since both the detection principle based on SERS and the information visualization methods are entirely generic, the approach may be extended to other pesticides and contaminants, including real samples.

Acknowledgements This work was supported by FAPESP, CNPq, CAPES, INEO, and nBioNet (Brazil).

Open Access This article is distributed under the terms of the Creative Commons Attribution 4.0 International License (http://crea tivecommons.org/licenses/by/4.0/), which permits unrestricted use, distribution, and reproduction in any medium, provided you give appropriate credit to the original author(s) and the source, provide a link to the Creative Commons license, and indicate if changes were made.

\section{References}

1. Sanitária ANDV (2005-2010) Avaliação dos Projeto da cooperação Técnica Anvisa, OPAS/OMS. Brasilia-DF

2. Alloway BJ, Ayres DC (1997) Chemical principles of environmental pollution, 2nd edn. Blackie Academic Professional, Glasgow

3. Li R-H, Liu D-H, Yang Z-H, Zhou Z-Q, Wang P (2012) Vortexassisted surfactant-enhanced-emulsification liquid-liquid microextraction for the determination of triazine herbicides in water samples by microemulsion electrokinetic chromatography. Electrophoresis 33(14):2176-2183. doi:10.1002/elps.201200104

4. Coutinho CFB, Tanimoto ST, Galli A, Garbellini GS, Takayama M, Amaral RB, Mazo LH, Avaca LA, Machado SAS (2005) Pesticidas: mecanismo de ação, degradação e toxidez. Pesticidas: Revista de Ecotoxicologia e Meio Ambiente 15:65-72

5. Pinto GMF, Jardim I (2000) Use of solid-phase extraction and high-performance liquid chromatography for the determination of triazine residues in water: validation of the method. J Chromatogr A 869(1-2):463-469. doi:10.1016/s0021-9673(99)01242-x

6. USEPA (1996) United States Environmental Protection Agency (USEPA). vol 2014

7. Lippolis MT, Concialini V (1988) Differential pulse polarographic-determination of the herbicides atrazine, prometrine and simazine. Talanta 35(3):235-236. doi:10.1016/0039-9140(88) 80072-9

8. Tolcha T, Merdassa Y, Megersa N (2013) Low-density extraction solvent based solvent-terminated dispersive liquid-liquid microextraction for quantitative determination of ionizable pesticides in environmental waters. J Sep Sci 36(6):1119-1127. doi: $10.1002 /$ jssc. 201200849

9. Nouws HPA, Delerue-Matos C, Barros AA, Rodrigues JA, Santos-Silva A (2005) Electroanalytical study of fluvoxamine. Anal Bioanal Chem 382(7):1662-1668. doi:10.1007/s00216-005$3310-5$

10. dos Santos LBO, Silva MSP, Masini JC (2005) Developing a sequential injection-square wave voltammetry (SI-SWV) method for determination of atrazine using a hanging mercury drop electrode. Anal Chim Acta 528(1):21-27. doi:10.1016/j.aca.2004. 10.008

11. Guse D, Bruzek MJ, DeVos P, Brown JH (2009) Electrochemical reduction of atrazine: NMR evidence for reduction of the triazine 
ring. J Electroanal Chem 626(1-2):171-173. doi:10.1016/j.jele chem.2008.12.006

12. Liu J, Hu Y, Zhu G, Zhou X, Jia L, Zhang T (2014) Highly sensitive detection of zearalenone in feed samples using competitive surface-enhanced Raman scattering immunoassay. J Agric Food Chem 62(33):8325-8332. doi:10.1021/jf503191e

13. Aroca R (2006) Surface-enhanced vibrational spectroscopy. Wiley, Chichester

14. Kneipp K (2007) Surface-enhanced Raman scattering. Phys Today 60:40-47

15. Willets KA, Van Duyne RP (2007) Localized surface plasmon resonance spectroscopy and sensing. Ann Rev Phys Chem 58:267-297. doi:10.1146/annurev.physchem.58.032806.104607

16. Le Ru EC, Etchegoin PG (2009) Principles of surface enhanced raman spectroscopy (and related plasmonic effects). Elsevier, Amsterdam

17. Lopes HJJ (2003) Garantia e Controle da Qualidade no Laboratório Clínico. Belo Horizonte-MG

18. Leopold N, Lendl B (2003) A new method for fast preparation of highly surface-enhanced Raman scattering (SERS) active silver colloids at room temperature by reduction of silver nitrate with hydroxylamine hydrochloride. J Phys Chem B 107(24): 5723-5727. doi:10.1021/jp027460u

19. Tejada E, Minghim R, Nonato LG (2003) On improved projection techniques to support visual exploration of multi-dimensional data sets. Inf Vis 2:218-231

20. Minghim R, Paulovich FV, Lopes ADA (2006) Content-based text mapping using multi-dimensional projections for exploration of document collections. In: Erbacher RF, Roberts JC, Grohn MT, Borner K (eds) Visualization and data analysis 2006, vol 6060. Proceedings of SPIE. doi: $10.1117 / 12.650880$

21. Cai J, Elrassi Z (1992) Micellar electrokinetic capillary chromatography of natural solutes with micelles of adjustable surfacecharge density. J Chromatogr 608(1-2):31-45. doi:10.1016/00219673(92)87103-f

22. Furini LN, Sanchez-Cortes S, López-Tocón I, Otero JC, Aroca RF, Constantino CJL (2015) Detection and quantitative analysis of carbendazim herbicide on Ag nanoparticles via surface-enhanced Raman scattering. J Raman Spectrosc 46(11):1095-1101. doi: $10.1002 /$ jrs. 4737

23. Moskovits M (1985) Surface-enhanced spectroscopy. Rev Mod Phys 57(3):783-826. doi:10.1103/RevModPhys.57.783

24. Bonora S, Benassi E, Maris A, Tugnoli V, Ottani S, Di Foggia M (2013) Raman and SERS study on atrazine, prometryn and simetryn triazine herbicides. J Mol Struct 1040:139-148. doi:10. 1016/j.molstruc.2013.02.025

25. Benassi E, Di Foggia M, Bonora S (2013) Accurate computational prediction of the structural and vibrational properties of s-triazine derivatives in vacuo. A DFT approach. Comput Theor Chem 1013:85-91. doi:10.1016/j.comptc.2013.03.010

26. Paulovich FV, Moraes ML, Maki RM, Ferreira M, Oliveira ON $\mathrm{Jr}$, de Oliveira MCF (2011) Information visualization techniques for sensing and biosensing. Analyst 136(7):1344-1350. doi:10. 1039/c0an00822b

27. Oliveira ON Jr, Pavinatto FJ, Constantino CJL, Paulovich FV, de Oliveira MCF (2012) Information visualization to enhance sensitivity and selectivity in biosensing. Biointerphases 7(1-4):53. doi:10.1007/s13758-012-0053-7

28. Aoki PHB, Carreon EGE, Volpati D, Shimabukuro MH, Constantino CJL, Aroca RF, Oliveira ON Jr, Paulovich FV (2013) SERS mapping in Langmuir-Blodgett films and single-molecule detection. Appl Spectrosc 67(5):563-569. doi:10.1366/12-06909
29. Rubira RJG, Camacho SA, Aoki PHB, Maximino MD, Alessio P, Martin CS, Oliveira ON Jr, Fatore FM, Paulovich FV, Constantino CJL (2014) Detection of trace levels of atrazine using surface-enhanced Raman scattering and information visualization. Colloid Polym Sci 292(11):2811-2820. doi:10.1007/ s00396-014-3332-7

30. Aoki PHB, Alessio P, Riul A Jr, De Saja Saez JA, Constantino CJL (2010) Coupling surface-enhanced resonance Raman scattering and electronic tongue as characterization tools to investigate biological membrane mimetic systems. Anal Chem 82(9):3537-3546. doi:10.1021/ac902585a

31. Koeber R, Fleischer C, Lanza F, Boos KS, Sellergren B, Barcelo D (2001) Evaluation of a multidimensional solid-phase extraction platform for highly selective on-line cleanup and high-throughput LC-MS analysis of triazines in river water samples using molecularly imprinted polymers. Anal Chem 73(11):2437-2444. doi:10.1021/ac001483s

32. Djozan D, Farajzadeh MA, Sorouraddin SM, Baheri T, Norouzi J (2012) Inside-needle extraction method based on molecularly imprinted polymer for solid-phase dynamic extraction and preconcentration of triazine herbicides followed by GC-FID determination. Chromatographia 75(3-4):139-148. doi:10.1007/ s10337-011-2173-5

33. Oliveira Brett AM, Da Silva LA (2001) Validation of novel biosensors in real environment and food sample. Paper presented at the 1st Workshop on Evaluation, Athens, Greece, May

34. Djozan D, Ebrahimi B, Mahkam M, Farajzadeh MA (2010) Evaluation of a new method for chemical coating of aluminum wire with molecularly imprinted polymer layer. Application for the fabrication of triazines selective solid-phase microextraction fiber. Anal Chim Acta 674(1):40-48. doi:10.1016/j.aca.2010.06. 006

35. Tuzimski T (2012) Application of RP-HPLC-diode array detector after SPE to the determination of pesticides in pepper samples. J AOAC Int 95(5):1357-1361. doi:10.5740/jaoacint.SGE_ Tuzimski

36. Wang Y, Chang Q, Zhou X, Zang X, Wang C, Wang Z (2012) Application of liquid phase microextraction based on solidification of floating organic drop for the determination of triazine herbicides in soil samples by gas chromatography with flame photometric detection. Int $J$ Environ Anal Chem 92(14): 1563-1573. doi:10.1080/03067319.2011.564618

37. Djozan D, Ebrahimi B (2008) Preparation of new solid phase micro extraction fiber on the basis of atrazine-molecular imprinted polymer: Application for GC and GC/MS screening of triazine herbicides in water, rice and onion. Anal Chim Acta 616(2):152-159. doi:10.1016/j.aca.2008.04.037

38. Bakas I, Hayat A, Piletsky S, Piletska E, Chehimi MM, Noguer T, Rouillon R (2014) Electrochemical impedimetric sensor based on molecularly imprinted polymers/sol-gel chemistry for methidathion organophosphorous insecticide recognition. Talanta 130:294-298. doi:10.1016/j.talanta.2014.07.012

39. Valera E, Garcia-Febrero R, Isabel Pividori M, Sanchez-Baeza F, Marco MP (2014) Coulombimetric immunosensor for paraquat based on electrochemical nanoprobes. Sens Actuators B Chem 194:353-360. doi:10.1016/j.snb.2013.12.029

40. Lopez Rodriguez ML, Madrid RE, Giacomelli CE (2013) Evaluation of impedance spectroscopy as a transduction method for bacterial biosensors. IEEE Latin Am Trans 11(1):196-200. doi:10.1109/tla.2013.6502802 